\title{
MedienPädagogik
}

Zeitschrift für Theorie und Praxis der Medienbildung

www.medienpaed.com

ISSN 1424-3636

Themenheft Nr. 37: Medienpädagogik als Schlüsseldisziplin in einer mediatisierten Welt. Perspektiven aus Theorie, Empirie und Praxis Herausgegeben von Henrike Friedrichs-Liesenkötter, Lara Gerhardts, Anna-Maria Kamin und Sonja Kröger

\section{Von der Kinderserie Die Pfefferkörner bis zum Ego-Shooter Call of Duty}

\section{Internet-, Social Media- und Smartphone-Nutzung im Übergang von Kindheit zu Jugend}

Henrike Friedrichs-Liesenkötter und Friederike von Gross

\section{Zusammenfassung}

Der Beitrag stellt zwei qualitative Studien der Autorinnen vor, welche zum einen die Veränderung der (Online-)Mediennutzung im Übergang von später Kindheit zum Jugendalter fokussieren und zum anderen persönliche Deutungen, Umgangsweisen und Reflexionsprozesse des Medienhandelns sichtbar machen. Letztere bleiben in quantitativen Studien in der Regel unberücksichtigt, sodass ein qualitativer Zugang mittels Gruppendiskussionen mit Heranwachsenden gewählt wurde. Die Gruppendiskussionen mit Grundschulkindern (4. Klasse) zeigen, dass sich die im Durchschnitt Neunjährigen bereits Medien und deren Inhalten, die sich an Jugendliche und Erwachsene richten, zuwenden. Ihr Verständnis über das Internet im Allgemeinen und über Social Network Sites im Besonderen ist aber noch ungenau und oberflächlich. Die Nutzung findet zudem oftmals noch eingebunden in den familiären Kontext statt. Dies ändert sich mit dem Erwerb des ersten eigenen Smartphones. Dieses bekommen die Kinder in der Regel nach der vierten Klasse im Übergang zur weiterführenden Schule, wie die Gruppendiskussionen mit den Befragten aus der fünften, sechsten und siebten Klassenstufe zeigen. Im Hinblick auf die Herausforderungen im Umgang mit Internet, Social Media und Smartphones lässt sich resümieren, dass die Medienkompetenz der befragten Kinder im Laufe der Zeit zunimmt; Unsicherheiten bleiben aber bestehen. 


\title{
From the Kids TV Series Die Pfefferkörner to the First-Person Shooter Call of Duty. Internet, Social Media and Smartphone Use in the Transition from Childhood to Adolescence
}

\begin{abstract}
The article presents two qualitative studies by the authors, which focus on the changes regarding the (online) use of media from childhood to adolescence and make visible personal interpretations, ways of dealing and reflection processes of media action. The latter are generally not taken into account in quantitative studies, so that we chose a qualitative methodological approach with group discussions with adolescents. Group discussions with primary school children (4th grade) show that nine-year-olds (in average) are already turning to media (content) aimed primarily at young people and adults. However, their understanding about the Internet in general and social networking sites in particular is still inaccurate and superficial. In addition, the use often takes place in the family context. This changes with the receipt of their first smartphones. In general, the children get one after the fourth grade during the transition to secondary school, as shown by the group discussions with the respondents from the fifth, sixth and seventh grades. With regard to the challenges of dealing with the Internet, social media and smartphones, the media literacy of the children surveyed increases over time; uncertainties remain, however.
\end{abstract}

\section{Ausgangslage: Die Vorverlagerung jugendkultureller medialer Erfahrungen in die Kindheit}

Obwohl die allgemeinen Geschäftsbedingungen von Facebook, Instagram und Co. eine Nutzung in der Regel erst ab 13 Jahren, mitunter sogar ab 16 Jahren (WhatsApp) erlauben, nutzen auch Kinder Messenger-Apps und Social Network Sites (SNS), wie die KIM-Studie wiederholt aufgezeigt hat (Medienpädagogischer Forschungsbund Südwest 2013; 2015a; 2017; 2019). Angaben über eine Einbettung der medialen Nutzung in die kindlichen Lebensstrukturen (in Familie und Gleichaltrigengruppe), das damit 
einhergehende Mediennutzungsrepertoire und die kindliche Reflexion des eigenen Medienhandelns werden jedoch in den quantitativen KIM-Studien und weiteren Mediennutzungsstudien wie etwa der Kinder-Medien-Studie 2018 (Kinder-Medien-Studie 〈KMS〉 2018) nicht abgefragt. Zwar wird in den KIM-Studien der Kontakt mit unangenehmen Inhalten im Internet (z.B. Porno-, Gewalt- oder Horror-Szenen) berücksichtigt, aufgegriffen wird jedoch nicht, welche Strategien Kinder im Umgang damit entwickeln. Ebenso unklar bleiben die Konfrontation und der Umgang mit Cyberbullying oder welche Kenntnisse Kinder über SNS besitzen sowie welches Grundverständnis Kinder über das Internet im Allgemeinen haben. In zwei qualitativen Studien der Autorinnen zur Internet- und Social Media-Nutzung von Pre-Teens und Teens, die im Fokus dieses Artikels stehen, wurde diesem Forschungsdesiderat mittels folgender Forschungsfragen nachgegangen: Wie gestaltet sich die Internet- und Social Media-Nutzung ab dem späten Grundschulalter, u.a. im Hinblick auf die Einbettung in Familie und Peergroup? Wie verändern sich Nutzungsweisen und Verständnis der Internet- und Social Media-Nutzung im Übergang vom Kindes- ins Jugendalter?

Ausgangspunkt der vorliegenden Erhebungen waren die bis dato ausschliesslich quantitativ vorliegenden Daten zur kindlichen Nutzung von Social Communities im Internet. Laut der repräsentativen KIM-Studie 2012 (Medienpädagogischer Forschungsbund Südwest 2013, 40-41) waren 44 Prozent der 6- bis 13-Jährigen in Deutschland Mitglied einer Community, wobei mit ansteigendem Alter die Zahl der Nutzerinnen und Nutzer zunahm (6-7 Jahre: 5\% Nutzerinnen und Nutzer; 8-9 Jahre: 18\%; 10-11 Jahre: 41\%; 12-13 Jahre: 68\%). Der durchschnittliche Einstieg in eine Community erfolgte mit 10,4 Jahren gegen Ende der Grundschulzeit. Facebook lag mit 55\% (ebd.) unter den von Kindern genutzten Communities vorne. Laut der aktuellsten KIM Studie (Medienpädagogischer Forschungsbund Südwest 2019, 36) folgt heute hinter der dominierenden Nutzung von WhatsApp (70\% zumindest selten) immer noch Facebook an zweiter Stelle, auch wenn mit gut 36 \% der interneterfahrenen Kinder nur noch gut ein Drittel aller Kinder Facebook zumindest selten nutzt (15 \% täglich, 11 \% ein-/mehrmals pro Woche; zudem nutzen 32\% Snapchat, 26\% Instagram mindestens selten). Die quantitativen Daten zeigen eine zeitliche Vorverlagerung der 
Nutzung und auch des Besitzes digitaler Medien auf, die sich vom Kleinkindalter an fortsetzt (Medienpädagogischer Forschungsbund Südwest $2015 b, 8)$. Durch die digitale Mediennutzung im familiären Kontext als Ort der primären Medienerfahrung sind Kinder zunehmend in die Strukturen des Medienmarktes eingebunden (Theunert 2015) und nutzen auch solche Medieninhalte, die in der Vergangenheit einer älteren Zielgruppe zugesprochen wurden. Kleinkinder wachsen mit der selbstverständlichen Präsenz von Smartphones auf (Wiesemann, Eisenmann, und Fürtig 2015) und YouTube ist bereits seit Jahren die Lieblingswebsite der 6- bis 13-Jährigen (2014: 24 \%; 2018: 41\%) (Medienpädagogischer Forschungsbund Südwest 2015a, 34; 2019, 34). Diese Entwicklung wird sowohl durch die zunehmend mobile Verfügbarkeit von Mediengeräten als auch durch die mittels Touchscreen intuitive Bedienbarkeit solcher Geräte begünstigt (Aufenanger 2013, 7). Eine solche «selektive Beschleunigung» (Maschke 2013, 101) des Übergangs der Kindheit in die Jugend gilt nicht nur hinsichtlich Medienbesitz und -nutzung, sondern betrifft auch weitere vormals jugendspezifische Erfahrungen (Paus-Hasebrink und Kulterer 2014, 49; Maschke 2013, 95ff.). So untersucht Maschke (2013) in der Studie Jugend.Leben im Rahmen einer quantitativen Studie im Jahr 2012, in welchem Alter 16- bis 18-Jährige spezifische Erfahrungen zum ersten Mal gesammelt haben (z.B. erstes eigenes Taschengeld; Beenden von Kinderspielen; erstes eigenes Handy). Im Vergleich zu den Ergebnissen der Vorstudie aus 2001 (Zinnecker 2003) zeigt sich vor allem im Hinblick auf den Besitz eines Handys und das Beenden von Kinderspielen eine deutliche Vorverlagerung der Jugendphase in die Kindheit. So geben rückblickend mehr als die Hälfte der befragten Jugendlichen an, im Alter zwischen 9 und 10 Jahren mit den «Kinderspielen das erste Mal aufgehört» (Maschke 2013, 97) zu haben. Analog dazu stieg im gleichen Zeitraum der Besitz des ersten eigenen Handys deutlich an (ebd., 101). Das Handy stellt somit eine Art «'Sesam, öffne dich' für den Übergang in die Jugendphase dar» (ebd., 99).

Die zunehmenden medialen Erfahrungen von Kindern bedeuten weiter eine zunehmende Auflösung der in der Industriegesellschaft vormals klar abgegrenzten Kinder- und Erwachsenenwelt. Während in der Vergangenheit Kindheit durch Spielen und Lernen geprägt war und sich deutlich von der mit Arbeit verbundenen Erwachsenenwelt abgrenzte, ist diese klare 
Trennung aufgrund der heutigen kindlichen Nutzung «nationaler und internationaler Informationsnetzwerke» (Hengst 2014, 27), die auch zum Arbeitsbereich der Eltern gehören, nicht mehr gegeben. Inwiefern sich eine solche Vorverlagerung in der Übergangsphase von Kindheit zur Jugend im Hinblick auf Social Media-Nutzung zeigt, steht im Fokus der beiden qualitativen Studien der Autorinnen, die im Folgenden vorgestellt werden.

\section{Forschungsinteresse und Methode der qualitativen Studien}

\subsection{Internet- und Social Media-Nutzung von Kindern im späten Grundschulalter (Vierte Schulklasse)}

In der ersten explorativen qualitativen Studie der Autorinnen zur Internet- und Social Media-Nutzung von Kindern im späten Grundschulalter stand die SNS Facebook im Fokus. Zum Zeitpunkt der Erhebung in 2014 war Facebook das dominierende soziale Netzwerk, auch bei jüngeren Heranwachsenden. In Anbetracht der Ergebnisse der KIM-Studie (Medienpädagogischer Forschungsbund Südwest 2013, 36ff.), die damals bereits eine Nutzung für Kinder im Alter von sechs bis 13 Jahren aufzeigte, waren v.a. detailliertere Einblicke in das kindliche Nutzungsverhalten der OnlineCommunity von Interesse. Bewusst erfolgte die Entscheidung für ein qualitatives Forschungsdesign in Ergänzung zu den vorliegenden quantitativen Daten, um stärker subjektive Erfahrungen und Deutungen erheben zu können (Flick 2014).

Im Zeitraum von Juni bis September 2014 wurden sechs Gruppendiskussionen an drei Grundschulen in Nordrhein-Westfalen mit Kindern im Alter von neun bis zehn Jahren durchgeführt. Einbezogen wurden in die Studie in drei Gruppendiskussionen 11 Kinder, die angaben, Facebook zu nutzen und in ebenfalls drei Gruppendiskussionen 13 Kinder, die benannten, Facebook nicht zu nutzen.

Da sich Gruppendiskussionen mit Kindern in der Regel weniger selbstläufig gestalten als Gruppendiskussionen mit Erwachsenen, wurde den Diskussionen ein halbstandardisierter Leitfaden zugrunde gelegt (Vogl 
2015, 61ff.). Um den narrativen Anteil der kindlichen Gruppendiskussionsteilnehmenden zu erhöhen, wurde als Gesprächsstimulus ein von der Gesprächsleiterin für die Studie erstelltes Profil ihrer Person mit dem erdachten Namen «Marie Moser» verwendet. Das Profil sollte zur Auseinandersetzung mit bestimmten Gesprächsthematiken anregen (z.B. Datenschutz und Umgang mit Fotos). So war etwa die Privatadresse der Gesprächsleiterin angegeben oder ein Bild ihrer nackten jüngeren Schwester als Säugling sichtbar (vgl. Abb. 1).

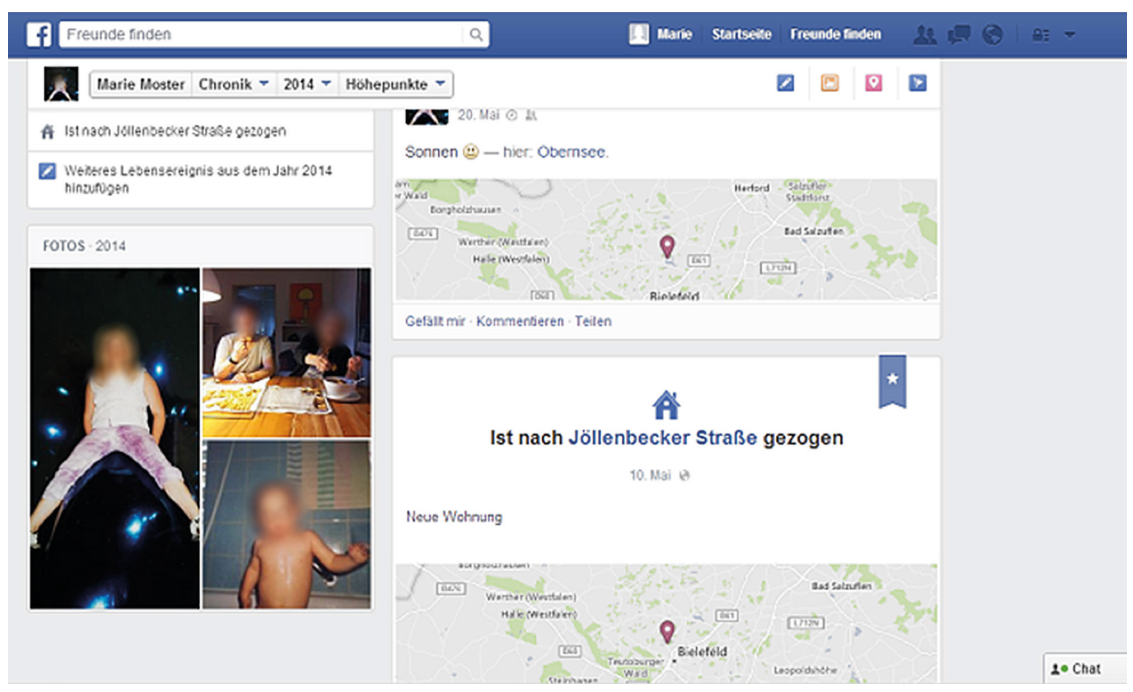

Abb. 1.: Stimulus: Fake-Profil der Gesprächsleiterin (Anm.: Gesichter nachträglich unkenntlich gemacht).

\subsection{Internet-, Social Media und Smartphone-Nutzung von Heranwachsenden der fünften, sechsten und siebten Klassenstufe}

Um zu erforschen, inwiefern sich im Übergang vom Kindes- zum Jugendalter Mediennutzungsweisen und das Verständnis für Strukturen und mögliche Risiken von Internet und Social Media verändern, wurden in der Folgestudie weitere Altersgruppen in den Blick genommen. Hierzu wurden sechs Gruppendiskussionen durchgeführt, jeweils zwei mit Schülerinnen und Schülern der fünften Klasse (Durchschnittsalter 11 Jahre), zwei 
mit Schülerinnen und Schüler der sechsten Klasse (Durchschnittsalter 12 Jahre) und weitere zwei mit Schülerinnen und Schüler der siebsten Klasse (Durchschnittsalter 13 Jahre). Als Stimulusmaterial wurden Logos von bekannten Social Media-Angeboten wie YouTube, WhatsApp oder Facebook genutzt. Der qualitative Leitfaden der ersten Studie wurde ergänzt, um jugendrelevante Medienpräferenzen entsprechend zu berücksichtigen und Erkenntnisse hinsichtlich einer möglichen Vorverlagerung jugendkultureller medialer Erfahrungen zu eruieren.

In beiden Studien erfolgte die Auswertung der Gruppendiskussionen durch eine Koppelung aus inhaltlich strukturierender und evaluativer qualitativer Inhaltsanalyse nach Kuckartz (2016).

\section{Zentrale Ergebnisse der Studien}

Im Folgenden werden die zentralen Ergebnisse beider Studien zunächst separat dargestellt. ${ }^{1}$

\subsection{Internet- und Social Media-Nutzung von Kindern im späten Grundschulalter (Vierte Schulklasse)}

Aus den Gruppendiskussionen mit den Schülerinnen und Schüler der vierten Klasse im Rahmen der ersten Studie liessen sich folgende Hauptergebnisse herausarbeiten:

\subsubsection{Verankerung der Facebook-Nutzung im familiären Kontext (Doing Family)}

Von den elf für die Studie ausgewählten Kindern, die sich selbst im Rahmen eines vorgeschalteten Kurzfragebogens als Facebook-Nutzerinnen und -Nutzer einordneten, geben in den Gruppendiskussionen lediglich fünf der Kinder an, über ein eigenes Profil zu verfügen, während die anderen Kinder das Profil ihrer Eltern zu nutzen scheinen. Es stellt sich heraus,

1 An dieser Stelle möchten sich die Autorinnen bei Anna Hübenet (erste Studie) und bei Bilsel Kiratli (zweite Studie) für die tatkräftige Unterstützung bei der Durchführung der Gruppendiskussionen und deren Analyse herzlich bedanken. 
dass lediglich zwei der fünf Kinder mit eigenem Nutzerkonto den Vorgang der Registrierung, die Umgehung der Altersbeschränkung sowie die Erstellung des eigenen Profils beschreiben können. Bei der Erstellung des Profils wurden die beiden Kinder - analog zu den Ergebnissen der KIMStudie 2014 (Medienpädagogischer Forschungsbund Südwest 2015a, 37) von einem Familienmitglied unterstützt.

Der Umstand, dass lediglich fünf der elf befragten Kinder der Gruppe der selbsternannten Nutzerinnen und Nutzer tatsächlich als FacebookNutzerinnen und -Nutzer mit eigenem Profil identifiziert werden konnten, weist darauf hin, dass es sich empfiehlt, Ergebnisse quantitativer Studien reflektiert zu lesen. Denn zumindest viele der im Rahmen der Studie befragten 9- und 10-jährigen Kinder konnten den Begriff Facebook-Nutzerin bzw. -Nutzer nicht derart definieren und anwenden. Es liegt also die Vermutung nahe, dass Kinder Fragen, die sie nicht im eigentlichen Sinne verstehen, diese entsprechend fehlinterpretieren und nicht im Sinne der Fragestellung beantworten können.

Eine zentrale Fragestellung der Studie war, inwiefern die FacebookNutzung im späten Grundschulalter noch in familiäre Nutzungsstrukturen eingebunden ist oder bereits einen Stellenwert im Kontext der sozialen Auseinandersetzung mit der Gleichaltrigengruppe einnimmt. Leitend war hierbei die Verortung der Schülerinnen und Schüler der vierten Klasse als so genannte Pre-Teens, die sich in einer Phase des Übergangs zum Jugendalter befinden, die durch eine beginnende Loslösung von den medialen Präferenzen der Eltern und einer Zuwendung zu den Vorlieben der Peergroup gekennzeichnet ist (Hoffmann 2012).

Die Auswertung zeigte, dass die kindliche Facebook-Nutzung der befragten Grundschülerinnen und -schüler fast ausschliesslich im Rahmen der Familie stattfindet und über gemeinsame genutzte Funktionen wie die Chat-Kommunikation kaum hinausreicht, wenngleich die Kinder viele Funktionen der Community benennen können, da sie diese bei ihren Eltern beobachten können. Nur eine Minderheit nutzt Facebook-Spiele oder schreibt Statusmeldungen. Fotos und Videos werden durch die Eltern oder ältere Geschwister auf deren Profil gepostet. Da es sich hierbei oftmals um Fotos gemeinsamer Familienaktivitäten handelt, welche die Eltern oder Geschwister auf ihrem Profil hochladen, scheinen sich die 
Grundschulkinder unmittelbar in deren Facebook-Nutzung eingebunden zu fühlen und demzufolge zum Grossteil nicht zwischen eigenen Posts und denen ihrer Familienmitglieder, vornehmlich der Eltern, differenzieren zu können. Die Beobachtung der Facebook-Aktivitäten anderer Familienmitglieder wird folglich in der subjektiven Konstruktion der Kinder zur wahrgenommenen eigenen Aktivität. Auch die begrenzte Einsicht der Kinder in die zugrundeliegenden Strukturen und Merkmale von Social Media-Anwendungen - z.B. dass eine Nutzung im Allgemeinverständnis mit einem eigenen Profil inkl. Nutzernamen, Passwort und weiteren Einstellungen verbunden ist - führt dazu, dass sie sich als Facebook-Nutzerinnen oder -Nutzer wahrnehmen.

Facebook besitzt im Rahmen der familiären Kommunikation der Kinder oftmals einen hohen Stellenwert. Vor allem bei denjenigen Kindern der Studie, die in Patchworkfamilien leben, bietet Facebook die Möglichkeit, niederschwellig mit vielen Familienmitgliedern Kontakt zu halten. So zählt etwa der 10-jährige Ben additiv alle diejenigen Familienmitglieder auf, die Facebook nutzen:

Ben: $\quad$ Mein Papa ist auf Facebook, meine Stiefmama ist auf Facebook [...], meine Tante ist auf Facebook, mein Onkel ist auf Facebook, mein anderer Onkel ist auf Facebook [...], meine Mutter ist auf Facebook (Pause), mein Stiefvater ist auf Facebook, meine andere Tante ist auf Facebook, [...] meine Schwester ist auch auf Facebook, [...] meine eine Cousine ist auf Facebook.

Darüber hinaus nutzen die Kinder im Rahmen der familiär verankerten Facebook-Nutzung die Möglichkeit, mit aufgrund von Globalisierungstendenzen über Landesgrenzen hinweg verteilten Familienmitgliedern zu kommunizieren. Facebook spielt dementsprechend eine bedeutende Funktion für das Doing Family, die Konstruktion familiärer Identität unter den Bedingungen von Entgrenzung und pluralisierten Familienformen (Theunert und Lange 2012, 18): Aufgrund von Wahlmöglichkeiten im Zuge der Entgrenzung der Geschlechterverhältnisse und der Entgrenzung von Familie (Tillmann und Hugger 2013, 37f.) sowie weiterer Individualisierungstendenzen ist es nicht mehr feststehende biografische Normalität, 
dass Familien generationenübergreifend im selben Wohnort leben, Kleinfamilien einer Gesamtfamilie im selben Land (d.h. Grosseltern, Tante/Onkel mit Partner*in und eventuellen Kindern) oder dass eine geschlossene Ehe lebenslang Bestand hat. Dies führt zu einem Wandel von Familie «von einer selbstverständlichen, quasi naturgegebenen Ressource zu einer zunehmenden voraussetzungsvollen Aktivität» (Theunert und Lange 2012, 12). Dementsprechend besteht ein familiärer Zusammenhalt nicht automatisch, sondern muss unter den gesellschaftlichen Bedingungen von Entgrenzung und Marktwirtschaft über ein Doing Family generiert werden, was Theunert und Lange (2012) als Herstellungsleistung von Familie verstehen. Diese Herstellungsleistung ist wiederum abhängig von materiellen, symbolischen, individuellen und gesellschaftlichen Ressourcen. Eine zentrale Ressource stellen in diesem Zusammenhang digitale Medien und vor allem die Kommunikationsmöglichkeiten des Internets dar. Per E-Mail oder Social Media-Nutzung können Familien bei räumlicher Trennung kommunizieren (Theunert und Lange 2012). Mit Blick auf die vorliegenden Studienergebnisse erleichtern SNS ähnlich wie andere internetbasierte Kommunikationsformen eine kostengünstige und direkte Kommunikation bei räumlichen Entfernungen, zum anderen bietet die Mediennutzung von Familienmitgliedern implizit eine «Anregungs- und Vorbildfunktion» (Theunert 2015, 136) für das kindliche Medienhandeln.

Bemerkenswert ist, dass Facebook laut der vorliegenden Studie innerhalb der Peergroup der Kinder kaum eine Rolle zu spielen scheint. Zwar geben einige Grundschülerinnen und -schüler an, Facebook zu nutzen, um mit weiter entfernten Freundinnen und Freunden zu kommunizieren, meist beschränkt sich die Kommunikation jedoch auf den familiären Kontext. Eine Facebook-Zugehörigkeit stellte somit zum Zeitpunkt der Erhebung keine in Bezug auf die Peergroup notwendige Bedingung für ein gelingendes Gruppengefüge dar. 


\subsubsection{Zuwendung von Kindern zu Medien(inhalten) für Jugendliche und Erwachsene}

Aus dem Kurzfragebogen der ersten Studie geht hervor, dass neben der familiären Internetnutzung der SNS Facebook 77 Prozent der befragten Kinder YouTube zu ihren Lieblingsseiten im Internet zählen. Hier rezipieren die Kinder verpasste TV-Sendungen, schauen zur Informationsrecherche gezielt Tutorials, z.B. zu den Themen Angeln und Häkeln, und nennen auch Lieblings-YouTuberinnen und -YouTuber wie z.B. Die Lochis oder Lieblingsformate wie Let's Plays.

Die kindlichen Aussagen in diesem Kontext und die ebenfalls in den Gruppendiskussionen wiederholt konstatierten medialen Besitzwünsche und Angaben zum medialen Eigenbesitz («Ich hab ein Handy!» «Ich kauf mir vielleicht bald ein Tablet!») hinsichtlich Smartphones und Tablets stützen die bereits mit Bezug auf andere empirische Studien festgestellte biografische Vorverlagerung.

Die Zuwendung von Kindern zu Medien und Medieninhalten, die sich eher an Jugendliche und Erwachsene richten, geht mit einer fortschreitenden Distanzierung gegenüber kindlichen Medieninhalten und-sendern einher. So grenzen sich bspw. Tim und Greta in ihren Äusserungen vom Internetportal des Fernsehsenders KiKA ab:

Greta: Kinderkram. [zeigt auf das KiKA-Logo]

I: Kinderkram?

Greta: Ich muss da manchmal drauf, wegen meiner kleinen Freundin.

Tim: Also ich habe ich war da nur drauf weil wegen so ner/ also auf KiKA/

Greta: Doch manchmal geh ich da drauf und gucke mir Schloss Einstein an, aber das mache ich auch manchmal auf YouTube/

Tim: Ich hab mir da mal ne Sendung für Grössere angeguckt, also so ab meinem Alter, wenn ich das verpasst habe.

Das Angebot des Senders KiKa wird als «Kinderkram» jüngeren Kindern zugewiesen. Wenn es genutzt wird, dann nur im Beisein jüngerer Freundinnen und Freunde sowie/ oder Geschwistern bzw. um sich gezielt 
Sendungen für ältere Kinder anzusehen, die aber statt auf KiKa auch gerne bei YouTube geschaut werden.

Einige Kinder benennen Strategien heimlicher Mediennutzung, um sich der Kontrolle der Eltern zu entziehen. So berichtet ein Kind von einer heimlichen Facebook-Nutzung, wenn die Eltern ausser Haus sind. Andere Kinder rezipieren bspw. Inhalte auf YouTube, die für Kinder nicht geeignet sind (z.B. Let's Play des mit einer USK-Alterskennzeichnung von 18 Jahren versehenen Computerspiels Grand Theft Auto) oder kaufen ohne vorherige Absprache im Internet ein.

Dies deutet auf ein beginnendes Aufbegehren einiger befragter Kinder gegenüber elterlichen Reglementierungen hin, während andere Kinder elterliche Vorgaben noch unhinterfragt übernehmen: Auf die, an die Nichtnutzerinnen und -nutzer gestellte Frage nach dem Wunsch, Facebook selbst nutzen zu wollen, benennen etliche Kinder mit Rückbezug auf elterliche Argumentationen, dass Facebook erst von älteren Kindern genutzt werden sollte und dass sie folglich eine eigene Facebook-Nutzung aufgrund ihres jungen Alters aktuell ablehnten.

\subsubsection{Problematische Online-Erfahrungen}

Alle Schülerinnen und Schüler der vierten Klasse der Studie können Risiken des Gebrauchs von SNS und der Internetnutzung im Allgemeinen wiedergeben. In diesem Zusammenhang umschreiben die Grundschülerinnen und -schüler verschiedene Schreckensszenarien wie eine sexuelle Annäherung durch Personen mit pädophilen Neigungen, Mord, Einbruch und Stalking, die bei einer unreflektierten Dateneingabe im Internet eintreffen könnten. Lisa (9 Jahre) erläutert dies wie folgt:

Wenn man zum Beispiel die richtige Adresse eingibt und den richtigen Namen, dann kann man die/ kann das auch ein Mörder oder so sein und man/ die können dich dann auch gut nach Hause/ zu dir kommen irgendwie (Pause) und was klauen oder so.

Als weitere Problematiken, die im Zusammenhang mit der Internetnutzung auftreten können, benennen die Kinder Computerviren, nicht absehbare Kosten, Hacking und Cyberbullying. Im Zusammenhang mit 
den genannten Risiken können sie auch Strategien zur Risikominimierung wiedergeben: Keine Datenpreisgabe von Telefonnummern, kein Posten von Nacktbildern und kein Annehmen fremder Dateien aufgrund von Virusgefahr. Bei auftretenden Problematiken sollte die Polizei gerufen und könnten kriminelle Personen geortet werden. Bislang beschränken sich die negativen Online-Erfahrungen der Kinder jedoch auf körperliche Reaktionen wie Kopfschmerzen infolge einer längeren Verweildauer, sodass die Kenntnisse über die genannten Risiken vermutlich eher aus medienerzieherischen Gesprächen mit den Eltern und/ oder einer Medien-AG an der Grundschule resultieren.

\subsection{Internet-, Social Media und Smartphone-Nutzung von Heranwachsenden der fünften, sechsten und siebten Klassenstufe}

In der Folgeerhebung mit den Schülerinnen und Schüler der fünften, sechsten und siebten Klasse wird die zunehmend grössere Bedeutung digitaler Medien für die Heranwachsenden, insbesondere des Smartphones, und die Verankerung der Social Media-Nutzung im Peergroup-Kontext deutlich.

\subsubsection{Das Smartphone als Statussymbol mit einer hohen emotionalen Verbundenheit}

Bemerkenswert ist, dass alle befragten Schülerinnen und Schüler des Samples bereits über ein eigenes Smartphone verfügen, das sie in ihrer Freizeit intensiv nutzen wie etwa die 11-jährige Claudia, die bei schlechtem Wetter drei Stunden mit dem Smartphone spielt, bis ihre Eltern es an sich nehmen. Das erste Smartphone erhielten die Schülerinnen und Schüler jeweils in der vierten oder fünften Klasse, teilweise weisen bereits Schülerinnen und Schüler der fünften Klasse eine «Handy- bzw. Smartphone-Biographie» auf, wie Claudia, die bereits ihr fünftes Handy/Smartphone besitzt. Die Schülerinnen und Schüler besitzen grösstenteils hochpreisige Geräte wie die zum Zeitpunkt der Erhebung angesagten Smartphones wie das iPhone 4s, 5 s oder das Samsung Galaxy S5. Diese bekommen sie in den meisten Fällen entweder zum Geburtstag oder zu Weihnachten geschenkt oder sie 
<erben> die Geräte von den Eltern, wenn diese ein neues Smartphone erhalten. Insgesamt ist ein stark ausgeprägtes Markenbewusstsein auszumachen, was sich vor allem in dem Wunsch nach einem iPhone manifestiert. Bereits die Schülerinnen und Schüler der fünften Klasse unterscheiden zwischen hochwertigeren und weniger hochwertigen Marken und wissen, dass ältere Modelle weniger beliebt sind, was sich in den Zitaten der elfjährigen Fünftklässlerin Kristina widerspiegelt:

Ich hätte gerne ein neues Handy, aber ich will jetzt nicht unbedingt ein iPhone 6, sondern ich wäre auch zufrieden mit dem Handy, was meine Mutter im Moment hat, also als iPhone 5. Das würde ich ganz gerne haben, weil ich möchte kein Samsung mehr. Hatte ich genug.

Weiter betont Kristina, dass in ihrer Familie «halt das von iPhone 5 bis iPhone 6Plus eigentlich Standard» sei - familiärer hochpreisiger Medienbesitz scheint hier zusätzliche Besitzwünsche zu schüren. Ein iPhone wird zudem von den Schülerinnen und Schüler als enorm teures Produkt wahrgenommen. Die Schülerinnen und Schüler der fünften Klasse vergleichen teilweise die Anschaffung eines iPhones mit dem Kauf eines Autos der Luxusmarken Ferrari oder Porsche.

Zudem nehmen sie das Smartphone als etwas Privates wahr, das man Geschwistern oder Eltern nicht in die Hand gibt, vergleichbar mit einem Tagebuch. Entsprechend hoch kann auch die emotionale Verbundenheit mit dem Gerät sein wie etwa bei der 13-jährigen Kiara (6. Klasse), welche eine symbiosenhafte Beziehung von Mensch und Maschine beschreibt:

[...] ich bin Kiara. Ich habe auch ein Handy. Ich liebe mein Handy. Ich verbringe nur mit meinem Handy Zeit. Das ist wie eine Zwillingsschwester für mich. Mein Handy heisst sogar Ciara. Mit C geschrieben.

Eine emotionale Verbundenheit oder auch Abneigung gegenüber dem eigenen Smartphone und bestimmten Apps zeigt sich bei den Schülerinnen und Schüler in emotionalisierten Ausdrücken: So «hasst» bspw. die Sechstklässlerin Jenny Minecraft und findet die App «scheiße», während ihr Facebook viel bedeutet: «Facebook, liegt mein Herz». 
Wenngleich das Smartphone und damit einhergehende Medienformate für die Schülerinnen und Schüler eine zentrale Rolle spielen, beschreiben diese eine Mediatisierung des Alltags, die sie durchaus kritisch betrachten. So bereuen sie teilweise, wenn sie Medienangebote aus ihrer Sicht zu lange genutzt haben, anstatt das gute Wetter zu geniessen oder mit Freundinnen oder Freunden etwas zu unternehmen. In diesem Zusammenhang nehmen sie durchaus auch zeitliche Restriktionen durch das Elternhaus als positiv wahr. So findet die Siebtklässlerin Lynn «die Regelung eigentlich ganz gut, dass man auch mal irgendwie nicht nur am Handy sitzt, sondern auch mal Zeit so zusammen verbringt.» Gerade im Hinblick auf Feste oder Geburtstage findet sie «Leute, die dann die ganze Zeit an ihrem Handy sitzen, [...] auch relativ doof, weil man trifft sich dann schon zu allen und dann kann man was zusammen machen und dann immer nur diese Leute, die an ihren Handys sitzen.» Dennoch findet - vor allem bei den Schülerinnen und Schüler der sechsten Klasse der Studie - eine heimliche Mediennutzung, teilweise sogar bis in die Nacht hinein, statt.

\subsubsection{Zwischen Kindheit und Jugend - Von den «Pfefferkörnern» bis zu «Call of Duty»}

Auch in der Erhebung mit den Schülerinnen und Schüler der weiterführenden Schulen zeigen sich Distinktionsbemühungen gegenüber Medieninhalten und -formaten, die sich an eine jüngere Zielgruppe richten. Zwar rezipieren sie noch hin und wieder Kindersendungen wie die KinderkrimiSerie «Die Pfefferkörner» der Öffentlich-Rechtlichen, aber auch Scripted Reality-Formate und explizite Inhalte für Erwachsene werden thematisiert. So schauen etwa die Schülerinnen und Schüler der fünften Klasse einer Gruppendiskussion auch YouTube-Videos der «Killer Clowns», in denen als Clowns verkleidete Menschen Morde nachstellen und Passantinnen und Passanten diese glaubhaft machen. Hierbei grenzen sich die Schülerinnen und Schüler deutlich von ihren jüngeren Geschwistern ab, da sie selbst diese Inhalte gut emotional verkraften würden:

Mira: Meine Schwester ne hat Albträume dadurch gekriegt.

Claudia: Tja, wir haben keine Albträume, wir sind/

Mira: Wir nich. Wir könnens. 
Zudem werden vereinzelt in der fünften Klasse neben altersgerechten Games wie Minecraft, über das kreative Welten gebaut werden können, auch gewalthaltige Games gespielt wie etwa der Egoshooter «Call of Duty». Bei den Schülerinnen und Schüler der fünften Klasse lässt sich folglich über ihre Mediennutzung rekonstruieren, dass sie sich in der Phase als Pre-Teen zwischen Kindheit und Jugend befinden.

\subsubsection{Beliebteste Apps}

Die beliebtesten Apps der Schülerinnen und Schüler sind WhatsApp, YouTube und Instagram, Facebook nutzen nur einige der befragten Schülerinnen und Schüler.

\section{WhatsApp}

WhatsApp als die primäre App zur Kommunikation der Schülerinnen und Schüler ist in der Regel die erste App, die auf das Smartphone geladen wird. Die App wird - analog zur Facebook-Nutzung der Schülerinnen und Schüler der vierten Klasse - im Rahmen des Doing Family genutzt. Darüber hinaus spielt die digitale Kommunikation mit der Peergroup ab der fünften Klasse eine zentrale Rolle, bspw. werden über WhatsApp Klassengruppen oder Gruppen für Freizeitaktivitäten wie etwa Fussball genutzt. Doch die Schülerinnen und Schüler beschreiben auch Nachteile der WhatsApp-Nutzung. So berichten die Schülerinnen und Schüler der fünften Klasse von einer überbordenden und damit auch belastenden Anzahl von 3.000 bis 10.000 WhatsApp-Nachrichten am Tag. Zudem sind einige Schülerinnen und Schüler bereits mit nervenden und mitunter auch verängstigenden digitalen Kettenbriefen in Berührung gekommen. Die Sechstklässlerin Laura berichtet hierbei von einer durch eine Computerstimme verzerrten Sprachnachricht, welche bei einem nicht Weiterversenden der Nachricht droht:

Laura: Ich hab mal sowas gekriegt. (Jan: heftisch) Das war auch so eine Computerstimme. Das war so ne Frau, die war so/ also verzehrte Computerstimme. Die hat so gesagt: «Wenn du das jetzt nicht an 15 Leute weiterschickst, dann bringe ich deine Mutter um. Frag, frag!» Da hat sie so/ also 
Beispiele ge/ Ich frage zum Beispiel: «Claudia [Name geändert]». «Die hab ich auch umgebracht, weil sie hat das nur // an drei Menschen//»

Tom: //Dann kannst du die auch fragen// (lacht)

(Jan lacht auch)

Laura (lachend): «an drei Leute weitergeschickt und hab ich sie umgebracht. Ihre Mutter, werde ich die halt irgendwie erst in drei Monaten umbringen» und so (lachend), keine Ahnung (...).

Zwar wissen die Schülerinnen und Schüler der sechsten Klasse prinzipiell, dass die Absenderinnen oder Absender der digitalen Kettenbriefe keinen Schaden anrichten werden, wenn man die Nachrichten nicht weiterschickt, das Erhalten der Nachrichten ist dennoch mit einem unangenehmen Gefühl verbunden, sodass sie diese weiterleiten.

YouTube

YouTube nimmt im Vergleich zur Erhebung mit den Schülerinnen und Schüler der vierten Klasse ab der fünften Klasse eine zentralere Rolle ein. Hier werden verschiedenste Channels und Video-Formate genutzt: Let's Plays, Beauty-Tutorials, Comedy-Clips, Challenges oder Videos aus dem aktuellen Fernsehprogramm, die zeitversetzt angeschaut werden. Die Schülerinnen und Schüler wissen um die wöchentlichen Veröffentlichungszeitpunkte der Videos ihrer Lieblings-YouTuberinnen und -YouTuber und sehen sich dann - wie beim klassischen Fernsehprogramm - zum entsprechenden Zeitpunkt die Videos an. Ab der sechsten Klasse werden zudem tendenziell auch Musikvideos angeschaut.

Instagram

Mit Blick auf Instagram und Facebook fallen altersspezifische Unterschiede in der Wahrnehmung der Apps auf, auch bezogen auf die Einschätzungen durch die Eltern der Schülerinnen und Schüler. Die Instagram-Nutzung ist den Pre-Teens durch ihre Eltern ab der fünften Klasse erlaubt, während die Facebook-Nutzung, vermutlich aufgrund negativer medialer Berichterstattung, nicht erlaubt wird. Entsprechend betrachten die Schülerinnen 
und Schüler der fünften und sechsten Klasse Facebook als eine App für Erwachsene, der sie verschiedene Risiken wie Cyberbullying, das Hacken des eigenen Accounts, das Auftauchen von unangemessenen Fotos sowie Stalking und sexuelle Belästigung zuschreiben, und aus diesem Grund Facebook auch nicht nutzen wollen. Dass bei Instagram dieselben Problematiken auftreten können, reflektieren die Schülerinnen und Schüler der fünften Klasse noch nicht ausreichend. In der siebten Klasse wandelt sich die Sicht auf Facebook: Nun wird die App abgelehnt, da es sich um eine App aus vergangenen Zeiten handele, welche die Schülerinnen und Schüler selbst nie genutzt haben und welcher sie im Vergleich zu Instagram auch keinen Mehrwert zuschreiben.

\subsubsection{Problematische Online-Erfahrungen und Medienkompetenz}

Neben den bereits angesprochenen digitalen Kettenbriefen benennen die Schülerinnen und Schüler der fünften und sechsten Klasse weitere negative Medienerfahrungen. So berichten drei von ihnen von Cyberbullying in einer WhatsApp-Gruppe oder von negativen Posts auf dem eigenen Facebook-Account. Zudem gehen fünf Schülerinnen und Schüler der fünften und sechsten Klasse davon aus, dass ihr Instagram- oder FacebookAccount bzw. ihr Computer gehackt worden sei, was sie beim Surfen im Internet gemerkt hätten. Hierbei scheinen die Schülerinnen und Schüler jedoch gelegentlich technische Probleme mit Hacking zu verwechseln (wie z.B. einen Systemabsturz oder Irritationen beim Posten auf mehreren eigenen Instagram-Accounts). Mit zunehmendem Alter nimmt das Wissen über Online-Risiken und Strategien, diesen zu begegnen, zu: Während die Schülerinnen und Schüler der fünften Klasse (hier durchschnittlich 11 Jahre alt) Online-Risiken zwar aufzählen können, aber die Möglichkeiten der Privatsphäreeinstellungen nicht kennen, nutzen die Schülerinnen und Schüler der sechsten Klasse ( $\varnothing 12$ Jahre) bereits diese Einstellungen. Die Schülerinnen und Schüler der siebten Klasse ( $\varnothing 13$ Jahre) kennen wiederum teilweise Fachbegriffe der digitalen Welt und wissen, dass die Kommunikation über Social Apps mittels einer End-zu-End-Verschlüsselung geschützt werden kann. 


\section{Zusammenfassung und Ausblick}

Zusammenfassend lässt sich festhalten, dass der Zugang zu Online-Medien - sei es im Rahmen der familiären Mediennutzung oder über das eigene Smartphone - für Kinder einen deutlich früheren Zugang zu Informationen und eine Erweiterung ihrer Kontakt- und ihrer Kommunikationsmöglichkeiten zulässt als dies für vorherige Kindergenerationen der Fall war. Mit den Vorteilen wachsen auch die Herausforderungen. Nicht immer ist es für die befragten Kinder leicht, die Komplexität der Strukturen im Netz und der eigenen Mediennutzung zu durchschauen. Vergleicht man die Ergebnisse der Gruppendiskussionen der Grundschülerinnen und -schüler der vierten Klasse mit denen der Schülerinnen und Schüler der unteren Klassen der weiterführenden Schulen, so wird deutlich, dass der erste Besitz eines eigenen Smartphones das Mediennutzungsverhalten der Kinder deutlich verändert. War bei den Schülerinnen und Schüler der vierten Klasse in den Gruppendiskussionen die Smartphone-Nutzung noch kein Thema und die SNS-Nutzung stark familiär eingebunden, so ist für die befragten Schülerinnen und Schüler ab der fünften Klasse der Besitz eines eigenen Smartphones von zentraler Bedeutung - vor allem innerhalb der Peergroup. Während die Schülerinnen und Schüler der vierten Klasse digitale Medien und Facebook vor allem im Rahmen eines Doing Family und meistens gemeinsam mit den Eltern nutzen, kommunizieren die Schülerinnen und Schüler der fünften, sechsten und siebten Klasse vor allem über WhatsApp: zwar auch mit ihren Eltern und Grosseltern, überwiegend aber mit ihren Freundinnen, Freunden sowie Klassenkameradinnen und -kameraden. Des Weiteren bestätigen die Ergebnisse die Abwanderungstendenz von Heranwachsenden von Facebook zu anderen Communities wie Instagram oder Snapchat, wie sie etwa auch die aktuellen JIM-Studien aufzeigen. So ist 2018 Facebook nur noch für 6\% der 12- bis 19-Jährigen eine der wichtigsten Apps (Medienpädagogischer Forschungsbund Südwest 2018, 36).

Während es kaum Unterschiede hinsichtlich der präferierten Apps im Verlauf von Klasse fünf bis sieben gibt, zeigt sich jedoch eine mit zunehmendem Alter anwachsende Medienkompetenz der Schülerinnen und Schüler, die sich in einem höheren Wissen über die Strukturen des Internets, mögliche Risiken und damit einhergehende Strategien zur Risikovermeidung (bspw. im Hinblick auf Hacking) widerspiegelt. 
Des Weiteren haben die von den Autorinnen befragten Schülerinnen und Schüler teilweise bereits negative Medienerfahrungen gemacht. Während es sich bei den Schülerinnen und Schüler der vierten Klasse noch um ausschliesslich körperliche Problematiken handelt wie Kopfschmerzen bei zu langer Computernutzungsdauer, berichten bereits Schülerinnen und Schüler der fünften und sechsten Klasse über Erfahrungen mit Cyberbullying. Die Dringlichkeit für eine begleitete Mediennutzung durch das Elternhaus und Aktivitäten der Medienbildung, z.B. über ausserschulische und schulische Projekte, wird hierdurch deutlich. Auch die teilweise heimliche Nutzung von nicht altersgerechten Inhalten, vor allem bei den Schülerinnen und Schüler der weiterführenden Schulen, zeigt eine solche Notwendigkeit auf, um potenziellen negativen Erfahrungen zu begegnen, die bei einer zunehmend aus dem elterlichen Kontext losgelösten Mediennutzung zu befürchten sind. Ziel ist es dabei, Heranwachsende zu begleiten, kompetente und selbstbestimmte Mediennutzerinnen und -nutzer zu sein, ihnen Strategien zu vermitteln und ggf. Hilfesysteme aufzuzeigen.

Als besonderer Wert der vorliegenden qualitativen Studie lässt sich festhalten, dass die für die Interpretation des Medienhandels wichtigen persönlichen Sichtweisen, Einschätzungen, Umgangsweisen und Reflexionsprozesse in den Gesprächen mit den Kindern und Jugendlichen sichtbar gemacht werden konnten. Möchte man zukünftig tiefergehende Ergebnisse zu diesen Aspekten erhalten, so bieten sich triangulative Verfahren aus quantitativen und qualitativen Methoden sowie detaillierte Operationalisierungen in quantitativen, Generalisierung ermöglichenden Studienanteilen, an. Bspw. könnten qualitative Interviews in Form von Portraits die quantitativen Daten bezüglich komplexerer Sachverhalte ergänzen oder Fragen zum Nutzungsverständnis in den quantitativen Studienteilen als Filterfragen vorgeschaltet werden. Zudem wäre es von Interesse, den soziodemografischen Hintergrund der Kinder einzubeziehen, was die qualitativen Studien der Autorinnen nicht leisten konnten. Aufschlussreich erscheinen zudem Durchführungen international vergleichender Analysen im Kontext der reflexiven Internet- und Social Media-Nutzung von Kindern, Pre-Teens und Teens. 


\section{Literatur}

Aufenanger, Stefan. 2013. «Neue Erfahrungsräume - neue Problembereiche. Aktuelle Herausforderungen für die Medienerziehung.» Medienconcret, Nr. 1:6-9.

Flick, Uwe. 2014. Qualitative Sozialforschung: eine Einführung. Reinbek bei Hamburg: rowohlts.

Hengst, Heinz. 2014. «Kinderwelten im Wandel». In Handbuch Kinder und Medien, herausgegeben von Angela Tillmann, Sandra Fleischer, und Kai-Uwe Hugger, 17-29. Digitale Kultur und Kommunikation, Band 1. Wiesbaden: Springer VS.

Hoffmann, Ole. 2012. «KidsReport 2011. Wie sieht die Realität des Kinderprogramms aus?» TELEVIZION, Nr. 1: 27-29.

Kinder-Medien-Studie (KMS). 2018. "Kinder-Medien-Studie 2018. Kinder in Deutschland: SMART! Mit und ohne Phone.» Kinder-Medien-Studie. https:// kinder-medien-studie.de/wp-content/uploads/2018/08/KMS2018_Berichtsband_v2.pdf.

Kuckartz, Udo. 2016. Qualitative Inhaltsanalyse: Methoden, Praxis, Computerunterstützung. Weinheim Basel: Beltz Juventa.

Maschke, Sabine, Hrsg. 2013. Appsolutely smart! Ergebnisse der Studie Jugend. Leben. Bielefeld: WBV, W. Bertelsmann Verlag.

Medienpädagogischer Forschungsbund Südwest. 2013. «KIM-Studie 2012. Kinder + Medien, Computer + Internet. Basisuntersuchung zum Medienumgang 6- bis 13-Jähriger». KIM-Studie. http://www.mpfs.de/fileadmin/KIM-pdf12/ KIM_2012.pdf.

Medienpädagogischer Forschungsbund Südwest. 2015a. «KIM-Studie 2014. Kinder + Medien, Computer + Internet. Basisuntersuchung zum Medienumgang 6- bis 13-Jähriger». KIM-Studie. http://www.mpfs.de/fileadmin/KIM-pdfi4/ KIM14.pdf.

Medienpädagogischer Forschungsbund Südwest. 2015b. «miniKIM 2014. Kleinkinder und Medien». miniKIM-Studie. http://www.mpfs.de/fileadmin/miniKIM/2014/miniKIM_2014.pdf.

Medienpädagogischer Forschungsbund Südwest. 2017. «KIM-Studie 2016. Basisuntersuchung zum Medienumgang 6- bis 13-Jähriger». KIM-Studie. https:// www.mpfs.de/fileadmin/files/Studien/KIM/2016/KIM_2016_Web-PDF.pdf.

Medienpädagogischer Forschungsbund Südwest. 2018. «JIM-Studie 2018. Basisuntersuchung zum Medienumgang 12- bis 19-Jähriger.» JIM-Studie. https:// www.mpfs.de/fileadmin/files/Studien/JIM/2018/Studie/JIM_2018_Gesamt. pdf.

Medienpädagogischer Forschungsbund Südwest. 2019. «KIM-Studie 2018. Basisuntersuchung zum Medienumgang 6- bis 13-Jähriger». KIM-Studie. https:// www.mpfs.de/fileadmin/files/Studien/KIM/2018/KIM-Studie2018_Web.pdf. 
Paus-Hasebrink, Ingrid, und Jasmin Kulterer. 2014. «Kommerzialisierung von Kindheit». In Handbuch Kinder und Medien, herausgegeben von Angela Tillmann, Sandra Fleischer, und Kai-Uwe Hugger, 47-57. Wiesbaden: Springer Fachmedien Wiesbaden. https://doi.org/10.1007/978-3-531-18997-0_3.

Theunert, Helga. 2015. «Medienaneignung und Medienkompetenz in der Kindheit». In Medienpädagogik - ein Überblick, herausgegeben von Friederike von Gross, Dorothee M. Meister, und Uwe Sander, 136-63. Weinheim und Basel: Beltz Juventa.

Theunert, Helga, und Andreas Lange. 2012. «Doing Family» im Zeitalter von Mediatisierung und Pluralisierung». merz medien + erziehung. Zeitschrift für Medienpädagogik 56 (2): 10-20.

Tillmann, Angela, und Kai-Uwe Hugger. 2013. «Mediatisierte Kindheit - Aufwachsen in mediatisierten Lebenswelten». In Handbuch Kinder und Medien, herausgegeben von Angela Tillmann, Sandra Fleischer, und Kai-Uwe Hugger, 31-45. Wiesbaden: Springer Fachmedien Wiesbaden. https://doi.org/10.1007/978-3531-18997-0_2.

Vogl, Susanne. 2015. Interviews mit Kindern führen: eine praxisorientierte Einführung. Grundlagentexte Methoden. Weinheim Basel: Beltz Juventa.

Wiesemann, Jutta, Clemens Eisenmann, und Inka Fürtig. 2015. "Medienpraxis in der frühen Kindheit». merz medien + erziehung. Zeitschrift für Medienpädagogik 59 (6): 39-47.

Zinnecker, Jürgen, Hrsg. 2003. Null zoff \& voll busy: die erste Jugendgeneration des neuen Jahrhunderts ; ein Selbstbild. 2., durchges. Aufl. Opladen: Leske + Budrich. 